\title{
Diagnóstico de TI en las MPyMES de la región Huasteca Hidalguense
}

\section{IT diagnosis in the SMEs of the Huasteca Hidalguense region}

\author{
FELIPE-REDONDO, Ana Maríał*, MENDOZA-SAN JUAN, Luis Alberto y SALAZAR- \\ CASANOVA, Hermes
}

Universidad Tecnológica de la Huasteca Hidalguense. Dirección de Tecnologías de la Información

ID $1^{\text {er }}$ Autor: Ana María, Felipe-Redondo / ORC ID: 0000-0002-8579-6532, Researcher ID Thomson: O-7111-2018, CVU CONACYT ID: 835952

ID $1^{\mathrm{er}}$ Coautor: Luis Alberto, Mendoza-San Juan / ORC ID: 0000-0002-7186-0177, Researcher ID Thomson: O-6650-2018, CVU CONACYT ID: 344988

ID $2^{\text {do }}$ Coautor: Hermes, Salazar-Casanova / ORC ID: 0000-0002-8444-6186, Researcher ID Thomson: O-6647-2018, CVU CONACYT ID: 926456

DOI: $10.35429 / J I T .2019 .19 .6 .15 .21$

Recibido: 10 de Marzo, 2019; Aceptado 30 de Junio, 2019

\section{Resumen}

El objetivo de este estudio es identificar las áreas de oportunidad para micro, pequeñas y medianas empresas (MPyMES) en la región huasteca del Estado de Hidalgo, que pueden optimizarse mediante el uso de las Tecnologías de la Información (TI). Se realizó una investigación exploratoria y descriptiva, con un enfoque cuantitativo. A través de la metodología Malhotra, se plantearon tres escenarios con respecto al uso de TI en las MPyMES. Si no la usan, ¿Por qué no las usan? Si usan TI, ¿en qué procesos los usan? ¿Qué otros procesos a través de TI pueden fortalecerse?, los resultados de la investigación revelan que algunas empresas no incorporan TI, porque consideran que su implementación es costosa, además no se tiene la experiencia para implementarla. Este estudio pretende, por un lado, identificar todas las áreas de oportunidad en el área de TI que presenten las MPyMES y, por otro lado, sirva esta información como un elemento de entrada al Cuerpo Académico CATI, para la gestión de proyectos que impacten en las microempresas, cumpliendo así el compromiso social que como grupo de investigadores tienen con la sociedad.

Diagnóstico TI, MPyMES, Malhotra

\begin{abstract}
Resumen
The objective of this study is to identify the areas of opportunity for micro, small and medium enterprises (MPyMES) in the Huasteca region of Hidalgo, which can be optimized through the use of IT. An exploratory and descriptive investigation was carried out, with a quantitative approach through the Malhotra methodology, Three ways were raised with respect to the use of ITs, the MPyMES. Why don't they use IT? If they use IT, in what processes do they use them? What other processes through IT can be strengthened?, the research results reveal that some of the MPyMES do not incorporate IT, because they consider that its implementation is expensive, additionally there is no experience to implement it.This study intends on the one hand, identifies all areas of opportunity in the area of ITs presented by MPyMES and on the other hand serves this information as an element of entry to the CATI Academic Body, for the management of their movements that have those aspects that impact on microenterprises, thus fulfilling the social commitment that as a group of researchers has with society.
\end{abstract}

ICT Diagnosis, MPyMES, Malhotra

Citación: FELIPE-REDONDO, Ana María, MENDOZA-SAN JUAN, Luis Alberto y SALAZAR-CASANOVA, Hermes. Diagnóstico de TI en las MPyMES de la región Huasteca Hidalguense. Revista de Tecnologías de la Información. 2019. 619: $15-21$

\footnotetext{
* Correspondencia del Autor (ana.felipe@uthh.edu.mx)

$\dagger$ Investigador contribuyendo como primer autor.
} 


\section{Introducción}

La Universidad Tecnológica de la Huasteca Hidalguense (UTHH), tiene actualmente 23 años de presencia en la región huasteca, su apertura ha sido un parteaguas en la región, a través de las diversas carreras pertinentes a la región y otras más bien, detonantes de procesos o dinámicas de trabajo que propicien una optimización, tal es el caso de las Tecnologías de la Información (TI).

El modelo educativo de la UTHH, favorece la vinculación entre escuela y sector productivo y social, a través de las estadías, las consultorías y particularmente, de los Cuerpos Académicos - como una función más del claustro docente - cuyo objetivo es impulsar la presencia de la ciencia que se practica en las universidades en la sociedad, atendiendo demandas locales con beneficios reales.

El Cuerpo Académico de Tecnologías de la Información (CATI) cuyo objetivo de su línea de investigación es asistir la sistematización de los procesos de las MPyMES de la región. $\mathrm{Su}$ estado actual es en formación y este ejercicio tiene como objetivo identificar áreas de oportunidad donde el CA pueda resultar de apoyo en el sector productivo y social de la región.

En la investigación bibliográfica se encontraron diversos estudios que documentan este cuestionamiento en diversas regiones del país, tal como lo mencionan en el artículo Los sistemas de información para las microempresas en México, los autores resaltan que el estudio les ha permitido "...darnos cuenta sobre el escaso conocimiento de muchos propietarios de microempresas, la falta de recursos y capacitación, la desigualdad en el acceso a las TIC y la falta de apoyo", (Lara Andrade, López Aguilar, \& Vázquez Juárez, 2018).

Este estudio resalta la situación de la región huasteca a fin de encontrar áreas de oportunidad para el CA y definir proyectos de investigación aplicada lo más cercanos a las necesidades reales del entorno. De acuerdo al Instituto Nacional de Estadística y Geografía (INEGI), en la región se tienen registradas 1,669 de micro, pequeñas y medianas empresas (INEGI, 2017), el estudio se enfoca en identificar cuáles son los principales usos de las TI, así como documentar aquellas MPyMES que aún no las implementan en sus procesos internos y sus motivos para no hacerlo.
Se describe como se determinó el universo así como el tamaño de la muestra de estudio a través de la Metodología Malhotra. Mención aparte merece la selección del instrumento de diagnóstico, así como la interpretación de resultados y los retos que como CA se presentan para impulsar el uso de las TI en las microempresas de la región. Por otro lado, si bien los resultados no distan mucho de otros obtenidos en otras regiones o sectores productivos, lo enriquecedor de este estudio es que permite conocer las demandas de apoyo del sector productivo y comercial de la región al que tendrán que encaminarse los esfuerzos del CA.

\section{Objetivo}

Identificar el grado de penetración de las TI en las MPyMES de la región Huasteca Hidalguense en los procesos internos, a fin de identificar áreas de oportunidad para su optimización.

\section{Marco Contextual}

\section{Clasificación de las MPyMES}

La clasificación publicada en el Diario Oficial de Federación (DOF) establece que el tamaño de la empresa se determinará a partir del obtenido del número de trabajadores multiplicado por $10 \%$; más el monto de las ventas anuales por $90 \%$, se describe en la Tabla 1.

\begin{tabular}{|c|c|c|c|c|}
\hline \multicolumn{5}{|c|}{ Estratificación } \\
\hline Tamaño & Sector & $\begin{array}{ll}\text { Rango } & \text { de } \\
\text { número } & \text { de } \\
\text { trabajadores } & \end{array}$ & $\begin{array}{l}\text { Rango de } \\
\text { ventas } \\
\text { anuales } \\
\text { (mdp) }\end{array}$ & $\begin{array}{l}\text { Tope máximo } \\
\text { combinado* }\end{array}$ \\
\hline Micro & Todas & Hasta 10 & Hasta $\$ 4$ & 4.6 \\
\hline \multirow[t]{2}{*}{ Pequeña } & Comercio & $\begin{array}{l}\text { Desde } 11 \text { hasta } \\
30\end{array}$ & $\begin{array}{l}\text { Desde } \\
\$ 4.01 \text { hasta } \\
\$ 100\end{array}$ & 93 \\
\hline & $\begin{array}{l}\text { Industria y } \\
\text { Servicios }\end{array}$ & $\begin{array}{l}\text { Desde } 11 \text { hasta } \\
50\end{array}$ & $\begin{array}{l}\text { Desde } \\
\$ 4.01 \text { hasta } \\
\$ 100\end{array}$ & 95 \\
\hline \multirow[t]{3}{*}{ Mediana } & Comercio & $\begin{array}{l}\text { Desde } 31 \text { hasta } \\
100\end{array}$ & \multirow{2}{*}{$\begin{array}{l}\text { Desde } \\
\$ 100.01 \\
\text { hasta } \$ 250\end{array}$} & \multirow[t]{2}{*}{235} \\
\hline & Servicios & $\begin{array}{l}\text { Desde } 51 \text { hasta } \\
100\end{array}$ & & \\
\hline & Industria & $\begin{array}{l}\text { Desde } 51 \text { hasta } \\
250\end{array}$ & $\begin{array}{l}\text { Desde } \\
\$ 100.01 \\
\text { hasta } \$ 250\end{array}$ & 250 \\
\hline
\end{tabular}

Nota: Diario Oficial de la Federación. (30 de 06 de 2009). Clasificación para las Micro, Pequeñas y Medianas Empresas (Mipymes)

Tabla 1 Clasificación de las empresas

FELIPE-REDONDO, Ana María, MENDOZA-SAN JUAN, Luis Alberto y SALAZAR-CASANOVA, Hermes. Diagnóstico de TI en las MPyMES de la región Huasteca Hidalguense. Revista de Tecnologías de la Información. 2019 


\section{Instrumento Diagnóstico}

La encuesta utilizada para elaborar el diagnóstico está basada en el estudio presentado por la National Small Business Association, una organización norteamericana que agrupa a las pequeñas empresas de ese país. Lo primero a cuestionar, sería ¿por qué utilizar un instrumento que no está enfocado al contexto nacional, y por ende muy despegado del ámbito regional?

Se compararon varios estudios tanto de ámbito nacional, tales como el instrumento que aplica el INEGI a través de la Encuesta sobre Tecnologías de la Información y las Comunicaciones, como de los países latinoamericanos, tales como la Encuesta de Acceso y Uso de TIC's en MicroEmpresas por parte del Gobierno de Chile y de Norteamérica, el grupo de investigadores concluyó que los aspectos que integra la encuesta cubren aquellas áreas de oportunidad que se pueden apoyar de acuerdo al perfil de los integrantes del CA, así como comparar con una economía con mejores condiciones que la nuestra, pero sobre todo basados en una visión de mejora.

Adicionalmente, se eliminaron y agregaron preguntas que se consideraron cubrían todos los aspectos que se buscan evaluar, además que el instrumento se sometió a validación de dos expertos externos de TI, cuyas opiniones permitieron hacer algunas adecuaciones y dar soporte al instrumento.

Las áreas que el instrumento de diagnóstico incluye son:

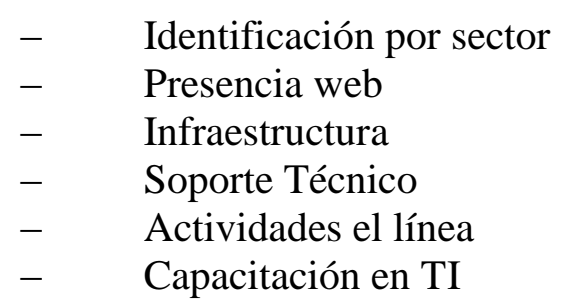

\section{Metodología a desarrollar}

Según Naresh A. Malhotra, "La Investigación de Mercados es la identificación, recopilación, análisis, difusión y uso, sistemático y objetivo de la información con el propósito de mejorar la toma de decisiones relacionadas con la identificación y solución de problemas y oportunidades de marketing", adecuando este proceso, se consideró una investigación de mercados, más bien para detectar las necesidades del mismo.
Las etapas de la metodología se describen a continuación:

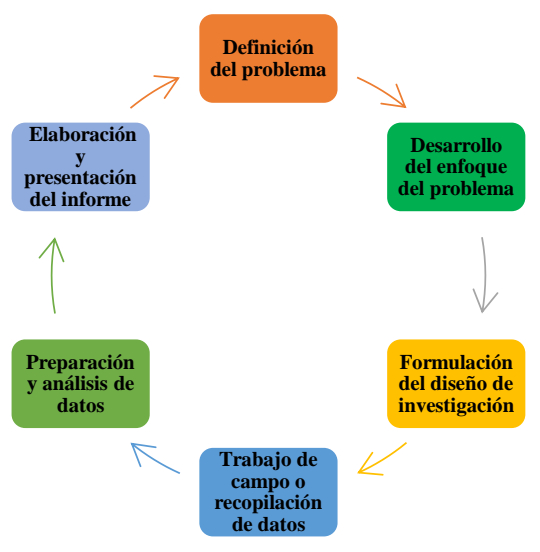

Figura 1 Secuencia de actividades de la Metodología Malhotra

\section{Definición del problema}

En esta etapa se define el contexto y el objetivo del proyecto de investigación, así como el análisis de los datos secundarios.

\section{Desarrollo del enfoque del problema}

En esta etapa se definió el marco de referencia, las preguntas de investigación y el planteamiento de la hipótesis.

\section{Formulación del diseño de investigación}

En esta etapa se define la información necesaria, se realiza el análisis de datos secundarios, se diseñó el cuestionario y el proceso de muestreo.

\section{Trabajo de campo o recopilación de datos}

Se realizó la selección, capacitación, supervisión y evaluación del equipo que operaría en el campo y se recopilaron los datos a través de cuestionarios.

\section{Preparación y análisis de datos}

Se verificaron los datos recopilados y se realizó el análisis de datos.

\section{Elaboración y presentación del informe}

Se redactó el informe y se informaron resultados. 


\section{Desarrollo}

\section{Definición del problema}

Si bien existe una gran cantidad de MPyMES en el estado de Hidalgo, no se ha realizado una investigación al respecto, particularmente en Huejutla, donde se registra según INEGI, 1669 microempresas.

\section{Desarrollo del enfoque del problema}

Preguntas de investigación

¿Por qué las MPyMES no hacen uso de las TIC? ¿Para que usan las MPyMES?

¿Para qué más podrían las MPyMES usar las TIC?

\section{Formulación del diseño de investigación}

La investigación tiene un enfoque cualitativo y exploratorio, haciendo uso del Directorio Estadístico de Unidades Económicas (DENUE) para documentar el universo de la investigación. En Huejutla de Reyes el total de MPyMES de la región es de 1669.

La encuesta incluyó 43 preguntas y se diseñó para solicitar la información de acuerdo a las áreas mencionadas anteriormente, las preguntas son mixtas, dicotómicas y abiertas. La encuesta se diseñó en Google Forms.

\section{Proceso de muestreo y tamaño de la muestra}

$\mathrm{N}$ : tamaño de la población 1669

Z: puntación 95:1.96

p: probabilidad de éxito: 0.50

q: nivel de confianza: 0.50

e: margen de error : 0.50

n:

$$
n=\frac{(Z)^{2}(P)(Q)(N)}{(e)^{2}(N-1)+(Z)^{2}(P)(Q)}
$$$$
n=\frac{(1.96)^{2}(0.5)(0.5)(1669)}{(0.05)^{2}(1669-1)+(1.96)^{2}(0.5)(0.5)}
$$$$
n=\frac{(3.8416)(0.5)(0.5)(1669)}{(0.0025)(1668)+(3.8416)(0.5)(0.5)}
$$$$
n=\frac{1602.9076}{10.215}
$$$$
n=312.43
$$

$n=312$

MPyMES.

\section{Trabajo de campo o recopilación de datos}

La aplicación de las encuestas se realizó donde geográficamente se da la mayor concentración de MPyMES.

Se hace uso de un dispositivo móvil para recabar las encuestas en un periodo de marzo junio de 2019. Se buscó la interacción de las entrevistas con los propietarios de las MPyMES a fin de tener la información de la fuente primaria.

\section{Resultados}

\section{Sector}
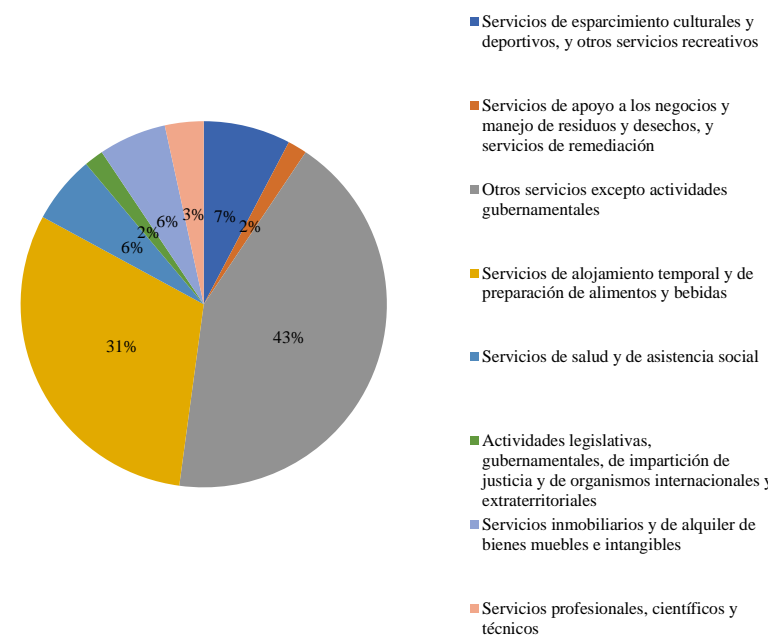

Figura 2 Distribución por sector

Los sectores más representativos son: el 43\% de las empresas pertenecen al sector de otros servicios, excepto actividades gubernamentales, el $31 \%$ corresponden al sector de servicios de alojamiento temporal y de preparación de alimentos y bebidas, el 7\% dedicadas a servicios de esparcimiento culturales y deportivos, en cuarto lugar se encuentra las dedicadas a los servicios de salud y los servicios inmobiliarios con el $6 \%$, en último lugar con el $2 \%$ se encuentra las empresas dedicadas a los servicios legislativos y de apoyo a negocios. 


\section{Presencia web}

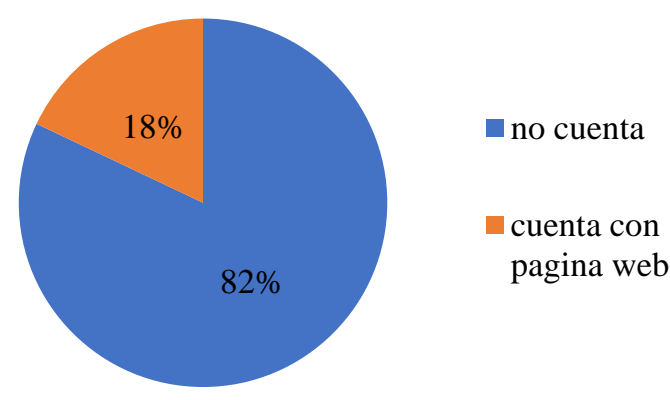

Figura 3 Distribución por presencia web

El $82 \%$ de los encuestados cuentan con una página web con la que dan a conocer sus productos al público por medio del internet, mientras que el $18 \%$ no cuentan con una página web.

\section{Infraestructura de TI}

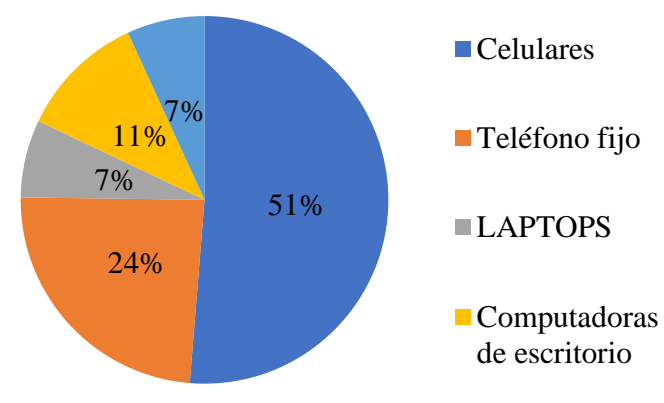

Figura 4 Distribución por Infraestructura de TI

Este gráfico muestra que el $51 \%$ de las empresas posee celulares que utilizan para hacer llamadas a clientes o proveedores, el $24 \%$ tiene por lo menos un teléfono fijo para realizar llamadas de negocios, también el $11 \%$ que poseen computadores de escritorio para trabajo de oficina o recolección de datos y con el $7 \%$ de empresas que tiene por lo menos un laptop o teléfono inteligente.

\section{Soporte Técnico}

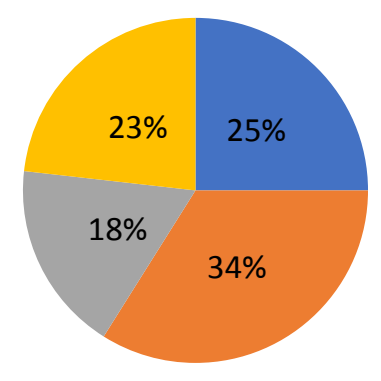

$$
\begin{aligned}
& \text { Paga a Expertos } \\
& \text { Técnicos Externos } \\
& \text { Usted mismo } \\
& \text { (Propietario) } \\
& \text { Familiares y } \\
& \text { amigos Técnicos } \\
& \text { Personal de la } \\
& \text { empresa }
\end{aligned}
$$

El 34\% de las empresas se encargan ellos mismos del soporte técnico de la infraestructura de TI, el $25 \%$ prefieren pagar a personal capacitado externo a la empresa para estas labores, también el $23 \%$ prefiere sus empleados se encarguen del soporte técnico y solo el $18 \%$ prefiere pedir ayuda a su familia o amigos que conozcan del tema.

\section{Actividades en Línea}

La actividad que más realizan las Pymes en línea es la compra a proveedores con un $38 \%$, el $25 \%$ es la publicidad que se realizan en línea, un $18 \%$ de las empresas encuestas no realizan ningún tipo de actividad en línea eso se manifiesta en la gráfica, con un porcentaje casi similar se encuentra el pago de nómina colocándose con un porcentaje del $15 \%$ y en último lugar se coloca la realización de llamadas o teleconferencias con un porcentaje del $4 \%$.

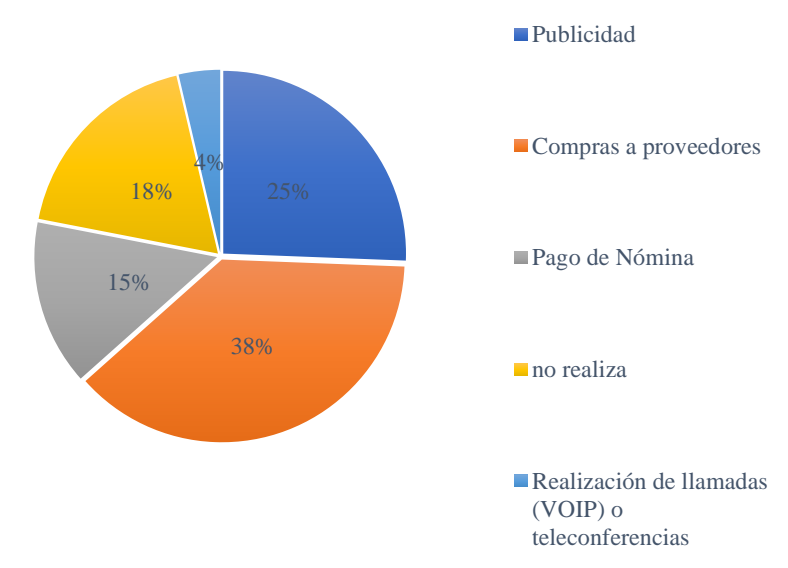

Figura 6 Distribución por Actividades en Línea

\section{Capacitación en TI}

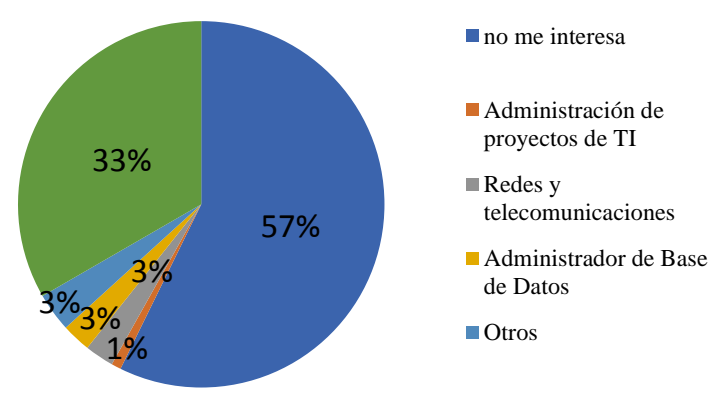

Figura 7 Distribución por Capacitación en TI 
La figura muestra que el $57 \%$ de las empresas no les interesa recibir capacitación en TI para su negocio, el $33 \%$ les importa capacitación sobre sistemas de información propios de su organización, con el 3\% a las empresas les interesa capacitar a su personal para administrar base de datos, redes y tecnologías y con el $1 \%$ le interesa la capacitación en la administración de proyectos de TI.

Los resultados por aspecto de la encuesta son los siguientes:

En relación a la Identificación por sector, se encontró que la mayoría son pequeños negocios de comercio y una menor porcentaje son servicios de hospedaje, dado que Huejutla es una zona turística por su riqueza cultural, por lo que desarrollar aplicaciones para este sector se presenta como un área de oportunidad.

Con relación a la Presencia web, si bien la mayoría cuenta con presencia web a través de páginas web, correo electrónico y redes sociales, su principal enfoque es de marketing y no te uso para procesos internos o comercialización, por lo que la formación como administrador de redes sociales se presenta como un área de oportunidad.

Por lo que hace a la Infraestructura, esta es pobre dado que sólo el $7 \%$ utiliza un equipo de cómputo para el control de procesos internos, este aspecto sin duda es uno de los que mayor impacto tienen, porque una de las razones que los participantes en la encuesta sostienen es el hecho de la inversión en TI es nula dado la inversión que representa, y la administración de las MPyMES es relativamente empírica.

El servicio de Soporte Técnico, refleja un $34 \%$ de MPyMES que cuentan con personal que atienden las necesidades internas, por lo que capacitación en esta área se presenta como una posible acción.

Las actividades de comercialización en línea son nulas y actualmente esta es el mayor nicho a desarrollar dado que se está dando auge a servicios de mensajería local, el impulso a aplicaciones móviles para comercialización sin duda es un aspecto que se demanda.
Por los que hace a la Capacitación en TI, los resultados se polarizaron porque por un lado la mayoría no desea invertir en capacitación de TI, pero quienes si lo desean hacen referencia a capacitación muy especializada.

\section{Agradecimiento}

A los miembros de la Cámara de Comercio Región Huejutla, quienes participaron de forma activa en este diagnóstico.

Al Dr. Efrén Juárez Castillo, docente de la Universidad Tecnológica de la Huasteca Hidalguense y al Dr. Lauro Vargas Ruiz, docente de Tecnológico de Oriente del Edo de Hidalgo, quienes amablemente revisaron $\mathrm{y}$ retroalimentaron el instrumento de diagnóstico.

\section{Conclusiones}

Por lo que dando respuesta a las preguntas de investigación

¿Por qué las MPyMES no hacen uso de las TIC? La mayoría de las microempresas encuestadas resalta altos los costos de inversión como un impedimento para su apropiación, así como la falta de conocimiento para su implementación, por lo que acciones de consultoría para algunas de ellas resulta una opción para definir sus prioridades.

¿Para que usan las MPyMES? La mayoría hace referencia a puntos de venta y servicios de marketing a través de redes sociales. las TIC?

¿Para qué más podrían las MPyMES usar

Como un elemento de apoyo a sus procesos internos así como soporte de comercialización en línea que contribuya a mejorar los ingresos de las microempresas de la región.

Un aspecto a resaltar es la falta de madurez de las empresas lo que les impide consolidar su presencia en el mercado y por ende en su crecimiento, optimizar sus procesos a través de las TI.

Sin duda este panorama le ofrece al CA diversas áreas de oportunidad a fin de propiciar en la región una adopción paulatina pero firme de los recursos de TI como una alternativa para mejorar los procesos de una microempresa.

FELIPE-REDONDO, Ana María, MENDOZA-SAN JUAN, Luis Alberto y SALAZAR-CASANOVA, Hermes. Diagnóstico de TI en las MPyMES de la región Huasteca Hidalguense. Revista de Tecnologías de la Información. 2019 


\section{Referencias}

Diario Oficial de la Federación. (30 de 06 de 2009). Clasificación para las Micro, Pequeñas y Medianas Empresas (Mipymes). Obtenido de Diario Oficial de la Federación: https://expansion.mx/emprendedores/2009/06/3 0/el-gobierno-reclasifica-las-pymes

INEGI. (2013). Encuesta sobre Tecnologías de la Información y las Comunicaciones (ENTIC 2013). Recuperada de http://www.inegi.org.mx/est/contenidos/proyect os/encuestas/establecimientos/otras/entic/defaul t.aspx

Lara Andrade, M., López Aguilar, G., \& Vázquez Juárez, P. (2018). Los Sistemas de Información para las microempresas en México. Horizontes de la Contaduría en las Ciencias Sociales, 5(9), 27-41. Obtenido de https://www.uv.mx/icp/files/2018/12/Num09-

Art03-153.pdf

Malhotra, , N. (s.f.). Investigación de Mercados (5ta. ed.). Prentice Hall.

Maldonado Guzmán, G., \& Martínez Serna, M., \& García Pérez de Lema, D., \& Aguilera Enríquez, L., \& González Adame, M. (2010). La influencia de las TICs en el rendimiento de la PyME de Aguascalientes. Investigación y Ciencia, 18 (47), 57-65. Recuperado de http://www.redalyc.org/html/674/67413393008/

Ministerio de Economía, Fomento y Turismo de Chile, (2006). Encuesta de Acceso y Uso de TIC's en MicroEmpresas. Recuperado de http://www.economia.gob.cl/estudios-yencuestas/encuestas/encuestas-deemprendimiento-y-empresas/encuesta-deacceso-y-uso-de-tics-en-microempresas

Münch, Lourdes, E. Á. (2009). Métodos y Técnicas de Investigación. México: Trillas.

National Small Business Association. (2013). 2013 Small Business Technology Survey. Obtenido de National Small Business Association: $\quad \mathrm{https}: / / \mathrm{www} . n s b a . b i z / w p-$ content/uploads/2013/09/Technology-Survey2013.pdf
Olmedo, Angélica, Vázquez, Edith, De León Irma \& Vanoye, Jorge. (2017) .Diagnóstico de las necesidades en la tecnología de información y comunicación en las Pymes de Actopan, Atitalaquia e Ixmiquilpan. Xikua, 5(9), 1-3. Recuperado de https://www.uaeh.edu.mx/scige/boletin/tlahuelil pan/n9/a1.html

Sánchez Abarca, J. (2019). La adopción de normas y modelos de calidad como detonante de la competitividad y de la transformación digital en las PyMES mexicanas de TI. Obtenido de Centro de Investigación e Innovación en Tecnologías de la Información y Comunicación: https://infotec.repositorioinstitucional.mx/jspui/ bitstream/1027/341/1/Adopcion_digital_en_PY MES_JSSA_2019_06_26.pdf

Sociedad para la Promoción y Reconversión Industrial. (2011). Guía de Autodiagnóstico para Pymes en la utilización de las TICs. Recuperado de https://es.slideshare.net/juandam10/gua-deauto-diagnostico-para-pyme-en-la-utilizacin-delas-tic 